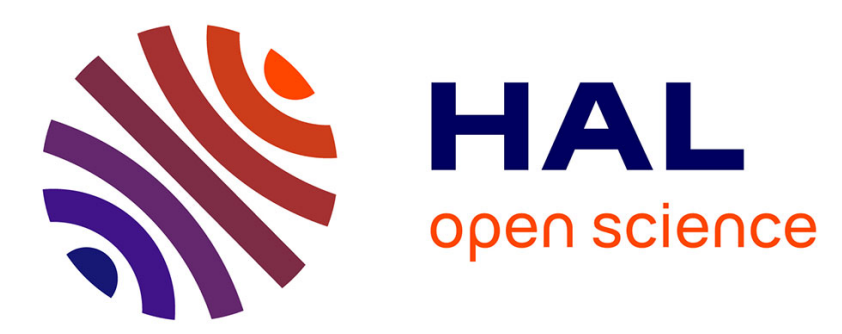

\title{
Approximations of functionals of some modulated-Poisson Voronoi tessellations with applications to modeling of communication networks
}

\author{
Bartlomiej Blaszczyszyn, René Schott
}

\section{- To cite this version:}

Bartlomiej Blaszczyszyn, René Schott. Approximations of functionals of some modulated-Poisson Voronoi tessellations with applications to modeling of communication networks. [Research Report] RR-5323, INRIA. 2004, pp.24. inria-00070677

\section{HAL Id: inria-00070677 \\ https://hal.inria.fr/inria-00070677}

Submitted on 19 May 2006

HAL is a multi-disciplinary open access archive for the deposit and dissemination of scientific research documents, whether they are published or not. The documents may come from teaching and research institutions in France or abroad, or from public or private research centers.
L'archive ouverte pluridisciplinaire HAL, est destinée au dépôt et à la diffusion de documents scientifiques de niveau recherche, publiés ou non, émanant des établissements d'enseignement et de recherche français ou étrangers, des laboratoires publics ou privés. 


\section{Approximations of functionals of some modulated-Poisson Voronoi tessellations with applications to modeling of communication networks}

Bartłomiej Błaszczyszyn — René Schott

$\mathbf{N}^{\circ} \mathbf{5 3 2 3}$

2004

THÈME 1 



\title{
Approximations of functionals of some modulated-Poisson Voronoi tessellations with applications to modeling of communication networks
}

\author{
Bartłomiej Błaszczyszyn*, René Schott ${ }^{\dagger}$ \\ Thème 1 - Réseaux et systèmes \\ Projet TREC
}

Rapport de recherche $\mathrm{n}^{\circ} 5323-2004-24$ pages

\begin{abstract}
We consider the Voronoi tessellation of Euclidian plane that is generated by an inhomogeneous Poisson point process whose intensity takes different constant values on sets of some finite partition of the plane. We show that mean functionals of a cell with the nucleus located in a given set of the partition can be approximated by the mean functionals of the typical cell of the homogeneous Poisson Voronoi tessellation with intensity appropriate to this partitioning set. We give bounds for the approximation errors, which depend on the distance of the nucleus to the boundary of the element of the partition it belongs to. In the case of a stationary random partition, we show that mean functionals of the typical cell of the respective double-stochastic Poisson-Voronoi tessellation admit an approximate decomposition formula. The true value is approximated by a mixture of respective mean functionals for homogeneous models, while the explicit upper bound for the remaining term, which depends on the covariance functions of the random partitioning elements, can be computed numerically for a large class of practical examples. This paper complements the previous studies in [9], where the distribution of the typical cell is approximated. One of the motivations for the study in question is modeling of modern communication networks, where application of the Poisson Voronoi tessellation has already proven to give some interesting results and where the assumption of the homogeneity is often non-adequate.
\end{abstract}

Key-words: Voronoi tessellation, modulated Poisson point process, double-stochastic Poisson point process, stochastic geometry, communication network, approximation, decomposability

\footnotetext{
${ }^{*}$ ENS/INRIA and Mathematical Institute University of Wrocław, ENS, 45 rue d'Ulm, 75005 Paris, France. E-mail: Bartek.Blaszczyszyn@ens.fr

$\dagger$ LORIA and IECN, Université Henri Poincaré-Nancy 1, BP 239, 54506 Vandoeuvre-lès-Nancy, France. E-mail: Rene.Schott@loria.fr
} 


\section{Approximation de fonctionnelles relatives aux mosaïques de Poisson-Voronoi modulées et applications en modélisation de réseaux de communication}

Résumé : Nous considérons la mosaïque de Voronoï du plan euclidien générée par un processus de Poisson ponctuel et inhomogène dont l'intensité prend différentes valeurs constantes sur les ensembles d'une certaine partition finie du plan. Nous montrons que des fonctionnelles moyennes d'une cellule dont le noyau est situé dans un ensemble donné de la partition, peuvent être approximées par les fonctionnelles moyennes de la cellule typique de la mosaïque de PoissonVoronoï avec une intensité appropriée à cet ensemble de la partition. Nous donnons des bornes pour les erreurs d'approximation qui dépendent de la distance entre le noyau et la frontière de l'élément de la partition correpondant à ce noyau. Lorsque la partition aléatoire est stationaire, nous obtenons des formules de décomposition approchée pour des fonctionnelles moyennes de la cellule typique de la mosaïque de Poisson-Voronoï doublement aléatoire. La valeur exacte est approximée par une mixture de fonctionnelles moyennes correspondant à des modèles homogènes. La borne supérieure du terme restant, qui dépend des fonctions de covariance des éléments de la partition aléatoire, peut être calculée numériquement pour une large classe d'exemples pratiques. Cet article complète une étude que nous avons effectuée précédemment concernant l'approximation de la distribution de la cellule typique. La modélisation des réseaux modernes de communication est une des motivations de notre travail. En effet l'utilisation des mosaïques de Poisson-Voronoï a d'ores et déjà permis d'obtenir des résultats intéressants dans ce domaine où l'hypothèse d'homogénéité est souvent non-adéquate.

Mots-clés : Mosaïque de Voronoï, processus de Poisson ponctuel modulé, processus de Poisson doublement aléatoire, réseau de communication, approximation, décomposabilité 


\section{Introduction}

Voronoi tessellation (VT) is a frequently used model of tessellation of the plane. For a given locally finite system of points (so-called nuclei) on the plane, the VT is a division of the plane into polygons "about" the points of the system. Precisely, the Voronoi polygon (cell in common terminology) about a chosen point of the system is the subset of points of the plane that lie closer to the chosen point than to any other point of the system. In modeling of communication networks this construction typically has the following interpretation: points denote locations of various structural elements (devices) of the network, while the cells denote regions of the plane served in some sense by these devices.

If the underlying system of points is a Poisson point process we call the resulting random tessellation the Poisson Voronoi tessellation (PVT). The PVT is often used in modeling of communication networks. The general idea of such stochastic approach consists in treating the given "real" ("observed") geometry of the network as a snapshot of a random model, and analyzing it in a statistical way. This allows for catching the essential spatial characteristics of the network through a minimum number of structural parameters (basically through the density of devices in the PVT case). This approach is especially useful for macroscopic economic analysis of the network.

In order to study statistical properties of random VT's one introduces the so-called typical cell of the tessellation. Very roughly speaking, in stationary case, it can be seen as "randomly chosen" from the set of cells. In non-stationary case its distribution depends on the location and is interpreted as conditional, given the underlying process has its point at this location (formal definitions require Palm theory of point processes).

The notion of the typical cell is essential for the stochastic geometry modeling of communication networks. Unfortunately, known formulae for distributional properties of the typical cell of PVT's are almost entirely confined to the stationary (homogeneous) case. Even then, formulae are very complicated and mainly approximations are known (see a review in Section 10.6 of [17]). Adopting homogeneous scenarios in communication models, however, is often too simplistic, since it ignores spatial fluctuations of the traffic (large cities versus rural areas etc). On the other hand, more adequate, non-homogeneous models rapidly become too difficult to analyze. A possible attitude to take if we want to improve upon this situation is to find a general framework, in which available results concerning homogeneous cases could be used as approximations in inhomogeneous cases.

In [9] the authors propose an approximation technique for the distribution of the typical cell of VT's generated by some class of modulated-Poisson point processes. The idea is to approximate the unknown distribution in the non-homogeneous case by a mixture of the known distributions for homogeneous Poisson cases. The authors give there analytically tractable bounds for the error of the approximation in total variation. In this paper we extend this approach studying mean functionals of the typical cell. This approach makes possible the analysis of a wide class of inhomogeneous PVT's by means of the formulae and estimates already established for homogeneous cases.

Specifically, we consider the Poisson point process whose intensity takes different constant values on sets of some finite partition of the space. Note that the cell of the VT about a given point is fully shaped by the neighbors of that point in the system of generating points. Thus, provided the partition of the space is not very "fine" with respect to the intensities of the points, the resulting modulated-Poisson Voronoi tessellation (mPVT) is "locally homogeneous" PVT. Consequently, the "typical cell of a given partitioning set" is highly probably identical to the 
typical cell of the homogeneous scenario and a "randomly chosen cell from the whole mPVT" should have a distribution close to the mixture of the homogeneous cases. The error of such approximation comes from existence of cells whose fundamental domain cross the boundaries between the partitioning sets. In the paper we formalize the above intuitive approximations and quantify the errors. Our analysis applies also to the Poisson process modulated by an independent stationary random partition, in which case the error of the approximation of the double-stochastic-Poisson VT depends on the so-called covariance function of the modulating partition.

One can find in our approach yet another incarnation of a general idea of decomposability. This technique, that has been productive in analysis of economic, queueing and computer system models, relies on clustering of a large system of all variables into a small number of groups so that: (i) the interactions among the variables of each single group may be studied as if interactions among groups did not exist, and (ii) interactions among groups may be studied without reference to the interactions within groups. The system in this case is completely decomposable. When the interactions between groups of variables are non-null, but weak compared with interactions within groups, the system is said to be near-completely decomposable (see the monograph [10]). Using this terminology, our mPVT is near-completely decomposable model (more precisely: "near-completely decomposable in mean").

The proposed macroscopic approximation technique applies to inhomogeneous PVT's which are piecewise (region-wise) homogeneous, provided this regionalization is not fine with respect to the density of nuclei. Inhomogeneous PVT's can be alternatively analyzed by space transformation technique or by simulation (see $\S 2.6$ in [5]).

The rest of the paper is organized as follows. First, in Section 2, we briefly scratch the idea proposed in [5] of stochastic modeling of the architecture of communication networks and explain how our results can contribute to this methodology. In Section 3 we introduce the Poisson Voronoi tessellation, functionals of its typical cell, and modulation schemes for its density. We discuss applications of these objects and schemes to modeling of communication networks. In Section 4, which is more mathematically oriented, we prove our main results concerning approximations of mean functionals of the typical cell of the modulated-Poisson Voronoi tessellation. These results are then discussed in their application context in Section 5, where some numerical examples are presented as well. Technical lemmas used in Section 4 are gathered in the Appendix.

\section{Stochastic modeling of the architecture of communica- tion networks}

In this section we briefly remind the idea, proposed in [5, 6], of stochastic modeling of the architecture of communication networks and explain how our results can contribute to this methodology.

\subsection{Modeling for large scale economic evaluation and strategic plan- ning}

The stochastic modeling of the architecure of a communication network seems relevant to large scale, economic evaluation and strategic planning of the network. Suppose for example that a new communication network is to be deployed or a given network is to be dimensioned so 
as to be able to serve a postulated traffic. This kind of task is usually subject to various cost-and-performance optimization analyses. In order to cope with such a complex problem in a mathematical way one needs to define a network model. This model should combine the following elements:

- description of the architecture of the network; i.e., the configuration of the geographical locations of various network devices (base station antennas and/or network controllers in cellular networks, concentrators in fixed telephony, access nodes in ad hoc networks, etc.),

- demand model; i.e, the amount of traffic and the way it is served by the network (number and repartition of users, call holding times, data traffic volume and repetition, etc.),

- cost, production and Quality of Service (QoS) functions; (cost of the deployment and the operation of the network, call blocking rates, effective throughput rates, etc.).

In the case of a dimensioning of a network, such a model should be first validated with respect to the real existing network. The architecture of the model should reflect the existing architecture and the values of the model functions calculated for the existing demand should correspond to these estimated in reality.

The strategic planning consists in finding a new model architecture that is able to serve a new demand (a forcast), and which is optimal in some way with respect to the cost production and QoS functions. More precisely one needs to specify a class of netwok architectures and to discriminate between them in order to choose an optimal one.

In the following we will describe possible attitudes one can take towards when modeling of the network architecture.

\subsection{Network architecture models}

We can distinguish the following ways of modeling of the network architecture.

\subsubsection{Deterministic architecture}

It consists in using a detailed geographical locations of existing and future planned network elements. The major shortcoming of this approach is that choosing between a few potential architectures needs tedious calculations of the model functions for each of them, and usually requires specific software optimization programs adapted to the specific type of architecture. An intrinsic methodological shortcoming of this approach is that it uses a considerable amount of parameters, out of which only some statistical characteristics are actually relevant for strategic planning. These statistical characteristics are not formally defined in the model or are difficult to identify when there are no closed-form analytical results connecting model parameters and functions.

\subsubsection{Homogeneous stochastic architecture}

It consist in treating the given existing and a future architecture of the network as a snapshot of a homogeneous random model, and analyzing it in a statistical way. In this approach, proposed in [5] (see also [6, 8]) and applied e.g. in [7, 1, 3, 4, 12, 2], the physical meaning of the network elements is preserved and reflected in the model, but their geographical locations are

$\mathrm{RR} \mathrm{n}^{\circ} 5323$ 


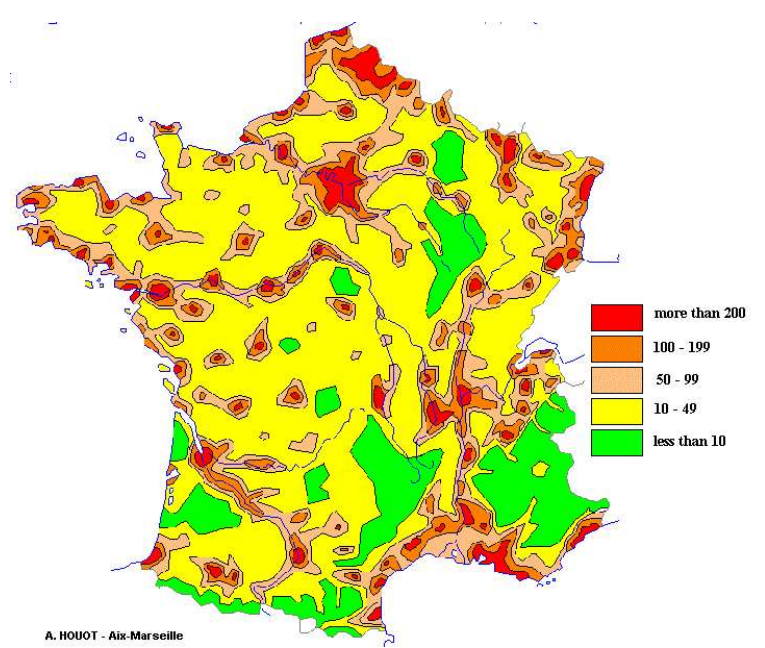

(a)

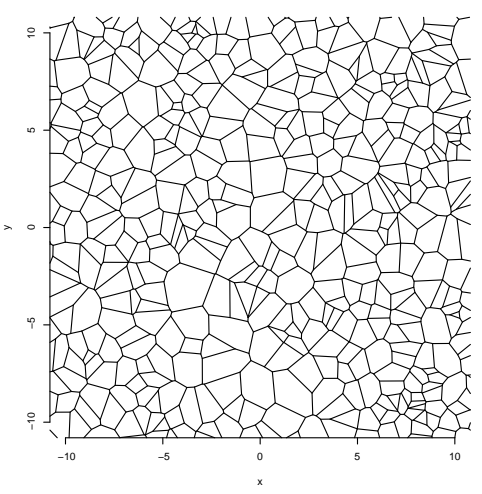

(b)

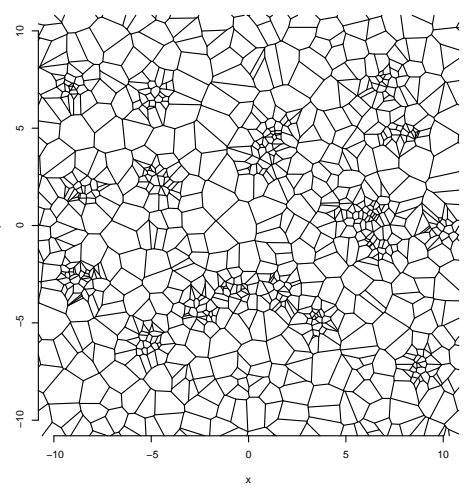

(c)

Figure 1: (a) Density of the population in France in 1990, in habitants $/ \mathrm{km}^{2}$, (b) a simulation of the homogeneous PVT tessellation, (c) a simulation of an inhomogeneous PVT, the inhomogeneity is modeled by taking the density of nuclei in 20 circular, randomly chosen regions of radius 1, to be 10 times bigger than in the remaining part of the plane (see PVT modulated by a Boolen model, Section 3.2.3)

no longer fixed but modeled by a random points of, typically, homogeneous planar Poisson point processes. Consequently, any particular detailed pattern of locations is no longer of interest. Instead, the method allows for catching the essential spatial characteristics of the network economy basically through the densities of these point processes (i.e., the densities of the network devices). Moreover, the cost and QoS functions, interpreted as the mean functionals of the respective Poisson-point-process driven models are often explicit functions of these densities. A simple but very useful example is Poisson-Voronoi tessellation.

Disputable aspects of this approach are the validation of a Poisson process as a statistical model of the localizations of the elements of a real network and the interpretation/implementation of the optimal Poisson-process based architecture. It is beyond the scope of this paper to discuss in details these questions. We remark only, that often, even architectures that are supposed to be very regular (eg. hexagonal cellular networks), in practice are very irregular due to various geographical positioning constraints. As far as the application of the optimal Poisson architecture is concerned, the results have to be interpreted in terms of the global densities of the network devices, with the detailed locations decided on empirical grounds.

\subsubsection{Inhomogeneous stochastic architecture}

The homogeneous-Poisson location of the network devices reflects various irregularities of a real network architecture. This irregularity is however homogeneous, meaning e.g. that the respective mean densities are constant on the plane. This assumption is often not very realistic. It is enough to give a look at a map of the density of population of a given region (see e.g. Figure 1) to realize that an optimal network that is supposed to reflect the traffic demand, should be inhomogeneous too.

Modeling of inhomogeneity is not an easy task. In to order preserve the advantages of the methodology described in $\S 2.2 .2$ one needs to propose simple parametric models of inhomo- 
geneous Poisson point processes. Moreover, for these models the respective mean functionals should be, at least approximately, expressed in explicit way in terms of the model parameters.

In the remaining part of this paper we will propose some simple class of inhomogeneous Poisson processes and will show how to approximate mean functionals of the Voronoi tessellation driven by them.

\section{Preliminaries}

In this section we introduce the Poisson Voronoi tessellation, functionals of its typical cell, and modulation schemes for its density. We discuss applications of these objects and schemes to modeling of communication networks.

\subsection{Homogeneous Poisson Voronoi tessellation and its typical cell}

\subsubsection{Voronoi tessellation}

For a given point process $\Phi=\left\{X_{i}\right\}$ on the plane $\mathbb{R}^{2}$, the Voronoi tessellation (VT) generated by $\Phi$ is the collection of convex sets $\left\{V_{i}\right\}$, where $V_{i}=V\left(X_{i}, \Phi\right)$ are sets (called also cells) of points in $\mathbb{R}^{2}$ that lie closer to the given point $X_{i}$ (called also the nucleus of $V_{i}$ ) than to any other point of $\Phi$; i.e.,

$$
V(x, \phi)=\left\{y \in \mathbb{R}^{2}:|y-x| \leq \inf _{\phi \ni z \neq x}|y-z|\right\}
$$

where $|x|$ is the Euclidian norm in $\mathbb{R}^{2}$ and $\phi=\left\{z_{i}\right\}$ is a collection of points in $\mathbb{R}^{2}$. If the underlying point process $\Phi$ is Poisson we will call $\left\{V_{i}\right\}$ the Poisson-Voronoi tessellation (PVT).

In modeling of communication networks, the VT might have the following interpretation:

- $\Phi=\left\{X_{i}\right\}$ denote locations of various structural elements (devices) of the network (base station antennas and/or network controllers in cellular networks, concentrators in fixed telephony, access nodes in ad hoc networks, etc.)

- $\left\{V_{i}\right\}$ denote regions of the plane served in some sense by the devices located respectively in $\left\{X_{i}\right\}$ (cells where telephone calls are carried by a given base station, domains of base stations connected to a given network controller, regions of fixed subscribers connected to a given concentrator, regions of ad hoc network served by a given access node, etc.)

\subsubsection{Typical cell of the PVT}

By the definition, the typical cell of a (stochastic) stationary Voronoi tessellation is a random set distributed as the cell of the nucleus located at 0 under so-called Palm distribution of the point process. It is considered as the distribution of the respective cell of the original Voronoi tessellation given the point process of nuclei has a point at 0 . In non-stationary context, the distribution of the typical cell depends on the location of its nucleus; i.e., the location of the conditioning point.

By Slivnyak's theorem (see Example 4.3 in [17]), the distribution of the typical cell with a nucleus located at $x$ in a general Poisson-Voronoi tessellation coincides with the distribution of the set $V(x, \Phi)-x=V(0, \Phi-x)$; i.e., with the distribution of the shifted to the origin cell 
created by an extra point at $x$ added to the original realization of $\Phi$. For more details on the Poisson-Voronoi tessellation see [15, 16] and in particular [14] for Palm methods.

The notion of the typical cell is essential for the stochastic geometry modeling of communication networks. Roughly speaking, in homogeneous scenario, the typical cell can be seen as a cell chosen at random, without any bias from the collection of all cells. Thus it is a random object, whose mean characteristics can be interpreted in terms of the following planar averaging: pick at random a (large) set of cells of the network, and take the empirical mean of their characteristics; then this empirical mean converges (for large samples of cells) to the mean characteristic of the typical cell (see [11]). In inhomogeneous scenario, this ergodic interpretation is more tricky; it would require, roughly speaking, sampling of cells from regions of the same density of points. Thus, it is more natural in this (inhomogeneous Poisson) context to think about the cell of an extra (test) point added to the original configuration.

We give now some examples of characteristics of the (typical) cell, naturally stemming in modeling of communication networks.

\subsubsection{Examples of cell functionals}

Let $V$ be a cell with the nucleus $X_{i} \in \Phi$. For simplicity suppose $X_{i}=0$. The following functionals of $V$ have been found relevant in modeling of communication networks.

- $\Psi(V)=|V|$, the area of $V$. Note that the following simple scenario leads to this functional. Consider a population of users living on $\mathbb{R}^{2}$ that is modeled by another, independent of $\Phi$, stationary Poisson (or general stationary) point process $\left\{Y_{i}\right\}$ with intensity $\mu$. Then the expected number of users in $V$ is given by $\mathbf{E}\left[\sum_{i} \mathbb{I}\left(Y_{i} \in V\right)\right]=\mu|V|$.

- $\Psi(V)=\int_{V} f(y) \mathrm{d} y$, the total cost or load of connecting of all points in $V$ to 0 , where $f$ is some non-negative cost function. Denote $S_{f}=\sum_{i} f\left(Y_{i}\right) \mathbb{I}\left(Y_{i} \in V\right)$, where $\left\{Y_{i}\right\}$ is as above. Then $\mathbf{E}\left[S_{f}\right]=\mu \int_{V} f(y) \mathrm{d} y$.

- $\Psi(V)=\int_{V} f(y) \mathrm{d} y \times \int_{V} g(y) \mathrm{d} y$; Define $S_{f}, S_{g}$, as above, for some non-negative cost functions $f, g$. Then $\operatorname{Cov}\left(S_{f}, S_{g}\right)=\mu^{2} \int_{V} f(y) \mathrm{d} y \times \int_{V} g(y) \mathrm{d} y+\mu \int_{V} f(y) g(y) \mathrm{d} y$.

- $\Psi(V)=|\partial V|$, the length of the boundary of $V$. Note that the following scenario, leads to this functional. Consider a system of roads on the plane modeled by another, independent of $\Phi$, stationary and rotation invariant Poisson point process $\left\{L_{i}\right\}$ on $\mathbb{R}_{+} \times[0, \pi)$, with intensity $\zeta$ (in other words, $\left\{L_{i}\right\}$ is a stationary and isotropic line process on the plane). Then the expected number of crossings of the boundary $\partial V$ of $V$ by roads $\left\{L_{r}\right\}$ is given by $2 / \pi \zeta|\partial V|$.

For more elaborate examples of cell functionals see e.g. [6, 3, 12]. Note that in all above formulas, we have considered the cell $V$ as a fixed (non-random) set, and all the expectations concern the distribution of either users or roads.

\subsubsection{Explicit formulas for mean functionals of the typical cell in homogeneous PVT}

Now we assume that $V$ is the (random) typical cell of the homogeneous PVT with intensity of points $\lambda$. We have the following explicit formulas for the mean functionals $\mathbf{E}[\Psi(V)]$ of the 
typical cell considered in Section 3.1.3

$$
\begin{aligned}
\mathbf{E}[|V|] & =\frac{1}{\lambda} \\
\mathbf{E}[|\partial V|] & =\frac{4}{\sqrt{\lambda}} \\
\mathbf{E}\left[\int_{V} f(y) \mathrm{d} y\right] & =\int_{\mathbb{R}^{2}} f(y) e^{-\lambda \pi|y|^{2}} \mathrm{~d} y, \\
\mathbf{E}\left[\int_{V} f(y) \mathrm{d} y \int_{V} g(y) \mathrm{d} y\right] & =\int_{\mathbb{R}^{2}} \int_{\mathbb{R}^{2}} f\left(y_{1}\right) g\left(y_{2}\right)\left[e^{-\lambda A\left(y_{1}, y_{2}\right)}-e^{-\lambda \pi\left(\left|y_{1}\right|^{2}+\left|y_{2}\right|^{2}\right)}\right] \mathrm{d} y_{1} \mathrm{~d} y_{2} .
\end{aligned}
$$

where $A\left(y_{1}, y_{2}\right)$ is the area of the union of two discs of radii $\left|y_{1}\right|,\left|y_{2}\right|$ centered in $y_{1}, y_{2}$; see e.g. Table 5.5.1 in [16] and [6].

\subsection{Modulated-Poisson Voronoi tessellations}

The homogeneity of the PVT is often not a very realistic assumption in modeling of communication network since it ignores spatial fluctuations of the density of traffic (large cities versus rural areas etc). On the other hand, more elaborate and adequate, non-homogeneous models rapidly become too difficult to analyze. We present here a simple approach allowing for modeling of spatial fluctuations of the Poisson intensity of points.

In the sequel, it will be useful to adopt the following point-measure notation: a given collection of points $\left\{x_{i}\right\}$ can be described by a counting measure $\phi$ on $\mathbb{R}^{2}$ defined by

$$
\phi(A)=\sum_{i=1}^{k} \varepsilon_{x_{i}}(A)
$$

for $A \subset \mathbb{R}^{2}$, where $\varepsilon_{x}$ is a Dirac measure with unit mass at $x$; i.e., $\varepsilon_{x}(A)=1$ if $x \in A$ and 0 otherwise. As a simple consequence of this notation we have for a given real valued function $f$ on $\mathbb{R}^{2}: \int f(x) \phi(\mathrm{d} x)=\sum_{i=1}^{k} f\left(x_{i}\right)$.

\subsubsection{Fixed modulation}

Let $\Phi_{u}=\left\{X_{i}\right\}=\sum_{i} \varepsilon_{X_{i}^{u}}, u=1, \ldots, \ell$ be independent stationary Poisson point processes on $\mathbb{R}^{2}$, with intensities, respectively $\lambda_{u}>0$. Let a measurable partition $\chi=\left\{\chi_{u}: u=1, \ldots, \ell\right\}$ of $\mathbb{R}^{2}$ be given. We call the inhomogeneous Poisson point process

$$
\Phi_{\chi} \equiv \sum_{u=1}^{\ell} \sum_{i} \mathbb{I}\left(X_{i}^{u} \in \chi_{u}\right) \varepsilon_{X_{i}^{u}}
$$

the $\chi$-modulated Poisson process $(\chi$-mod PP). Obviously $\chi$-mod PP is an inhomogeneous (in general) Poisson point process with intensity measure $\Lambda_{\chi}(\cdot)$ given by

$$
\Lambda_{\chi}(\mathrm{d} x) \equiv \mathbf{E}\left[\int_{\mathbb{R}^{2}} \mathbb{I}(y \in \mathrm{d} x) \Phi_{\chi}(\mathrm{d} y)\right]=\sum_{u=1}^{\ell} \mathbb{I}\left(x \in \chi_{u}\right) \lambda_{u} \mathrm{~d} x .
$$

The Voronoi tessellation

$$
\mathcal{V}\left(\Phi_{\chi}\right) \equiv\left\{V\left(X_{i}, \Phi_{\chi}\right): X_{i} \in \Phi_{\chi}\right\}
$$

generated by $\Phi_{\chi}$ will be called the $\chi$-modulated-Poisson Voronoi tessellation $(\chi$-mod PVT).

$\mathrm{RR} \mathrm{n}^{\circ} 5323$ 


\subsubsection{Random modulation}

We will also consider a stationary modulating random partition $\Xi=\left\{\Xi_{u}: u=1, \ldots, \ell\right\}$; that is, for any vector $x \in \mathbb{R}^{2}$ the distribution of $\Xi+x=\left\{\Xi_{u}+x: u=1, \ldots, \ell\right\}$ is the same as $\Xi$. Moreover, let $\Xi$ be independent of Poisson processes $\Phi_{u}, u=1, \ldots, \ell$. This makes the $\Xi-\bmod \mathrm{PP} \Phi_{\Xi}$ the stationary double-stochastic-Poisson (Cox) point process and the stationaryCox Voronoi tessellation $(\mathrm{CoxVT}) \mathcal{V}\left(\Phi_{\Xi}\right)$.

\subsubsection{Examples of modulations}

We will demonstrate here some examples of simple modulations in their possible contexts of applications.

- Cell located at some distance to a "hot spot". Assume $\chi_{1}=B_{(-r, 0)}(r)$, where $B_{x}(r)$ is the disc in $\mathbb{R}^{2}$ centered at $x$, with radius $r$, assume $\chi_{2}=\mathbb{R}^{2} \backslash \chi_{1}$ and consider a nucleus $x \in \chi_{2}$. Let $\lambda_{1}>\lambda_{2}$. This can be a simple model of the following scenario: consider a city (modeled by the disc) with much larger density $\lambda_{1}$ of some kind of communication devices (cf. Section 3.1.1), and consider a particular device located at $x$, outside the city, where the respective density is considerably smaller. Obviously, if the distance from this particular device to the city $(|x|$ in our example) is large, then the distribution, and hence all the mean functionals, of the cell served by the device located at $x$, is approximately the same as the distribution of the typical cell in homogeneous Poisson scenario with density $\lambda_{2}$. How

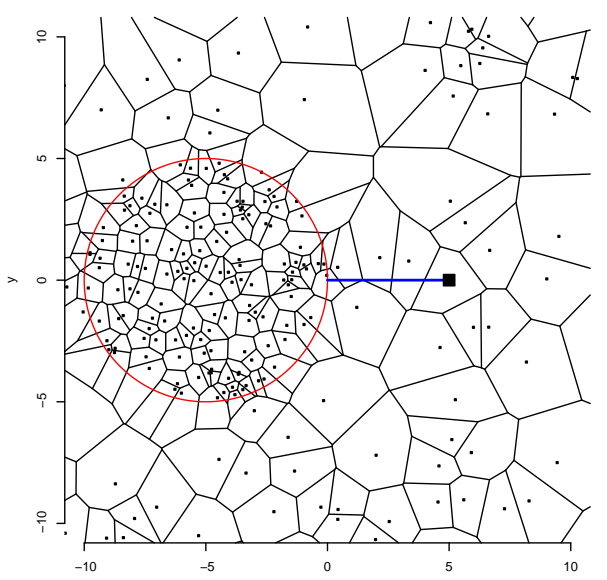

Figure 2: ${ }^{-10}$ Simulation of ${ }^{-5}$ a PVT with a hot spot, where the density of nuclei is 10 times bigger than outside it. good such an approximation is for a given distance $|x|$ is a natural question in this context. One can consider also a reverse situation, with $\lambda_{1}<\lambda_{2}$ (a "cold spot").

Cell located at some distance to a "hot wall". Let $\chi_{1}$ be a half-plane, $\chi_{2}=\mathbb{R}^{2} \backslash \chi_{1}$, and suppose $x \in \chi_{2}$, with $|x|$ being the distance of $x$ to $\chi_{1}$. Let $\lambda_{1}>\lambda_{2}$. This model is supposed to describe a similar scenario as previously, but with the region of the larger intensity of devices being so vast (comparing to the distance $|x|)$ that it is "seen" from $x$ as a half-plane. It seems to be relevant as well to a network deployed in a coastal region, where the population density is relatively large along the cost.

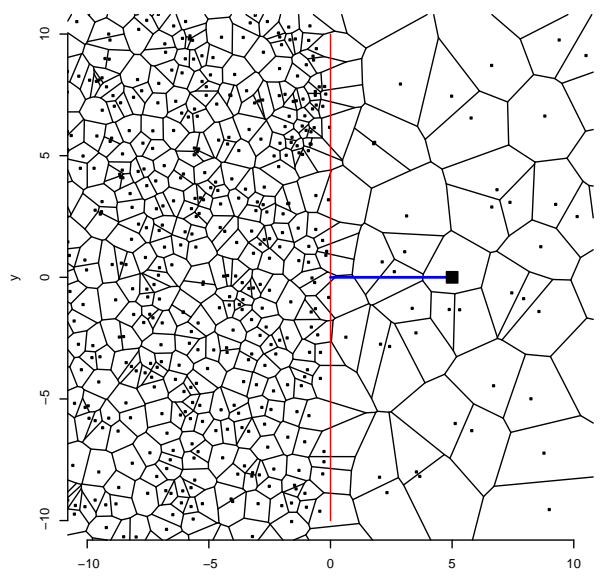

Figure 3: Simulation of a PVT with a hot wall, where the density of nuclei is 10 times bigger than elsewhere. 
- Typical cell of the PVT modulated by a Boolean model. Let the partition $\Xi=\left\{\mathbb{X}, \mathbb{X}^{c}\right\}$ be given, where $\mathbb{X}$ is a stationary Boolean model (BM); i.e.,

$$
\mathbb{X}=\bigcup_{i}\left(C_{i}+Y_{i}\right)
$$

where $\left\{Y_{i}\right\}$ is a Poisson point process (of the so-called germs) on $\mathbb{R}^{2}$ and $\left\{C_{i}\right\}$ is a sequence of (possibly random, independent, identically distributed) subsets of $\mathbb{R}^{2}$ (called grains). Let for example $\lambda_{1}>\lambda_{2}$. In order to demonstrate a possible application context of this model consider a country in which regions of a higher density of communication devices are scattered irregularly. Suppose we are not interested in a given particular location but in some "average device" typical for the whole country (such notion is useful e.g. for a global economical planning). Then, instead of analyzing the given "real" configuration of regions of higher density, it is customary to consider it as a snapshot of a (random) BM $\Xi$, where germs $\left\{Y_{i}\right\}$ model (e.g.) geographical centers of this regions and $\left\{C_{i}\right\}$ model regions themselves, centered to 0 . Now, provided the par-

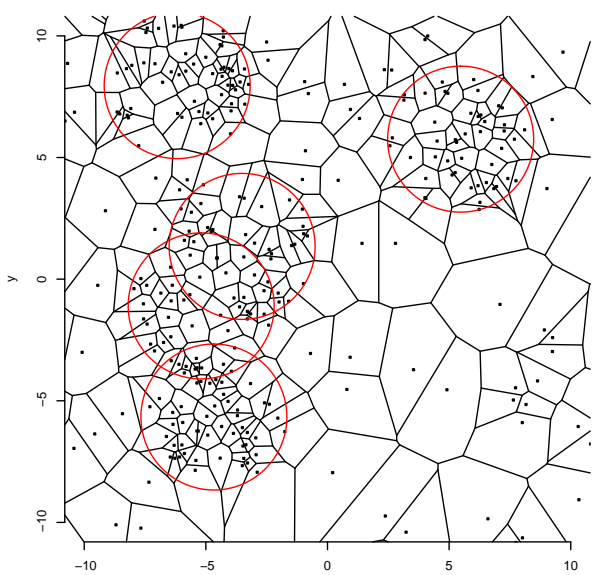

Figure 4: PVT modulated by a Boolen model: the inhomogeneity is modeled by taking the density of nuclei in 5 circular, randomly chosen regions of radius 3 , to be 10 times bigger than in the remaining part of the plane.

titioning of the plane by the BM is not very "fine" with respect to the densities of the devices, the typical cell for the whole country should have a distribution close to the linear combination of the homogeneous cases with densities $\lambda_{1}$ and $\lambda_{2}$, with the coefficients given by the fractions of the plane covered by the BM $\Xi$ and its complement, respectively. The error of such approximation, which we want to quantify, comes from existence of cells whose fundamental region cross the boundaries between the partitioning sets (see Section 4).

In the case of the above modulation schemes the mean functionals of the typical cell do not admit or admit very complicated formulas analogous to these presented in Section 3.1.4. However, sometimes the approximations suggested above can be easily constructed by means of the explicit formulas valid for homogeneous scenarios.

\section{Approximation results}

In this section we show how the mean functional of the typical cell of the modulated-Poisson Voronoi tessellation can be approximated by the respective means in homogeneous scenarios. In the case of the fixed modulation, the quality of the approximation depends on the (fixed) distance of this point to the boundary of the element of the partition it belongs to. In the case of a random modulating partition the quality of the approximation depends on the so-called covariance functions of the random sets of the partition. The mathematical results presented here are discussed in their application context in Section 5, where some numerical examples are presented as well.

We sketch first our basic idea how to identify a cell that is not typical for a given element of the partitioning $\chi$. By this we mean a cell $V\left(x, \Phi_{\chi}\right)$, with $x \in \chi_{u}$ for some $u \in 1, \ldots, \ell$, 


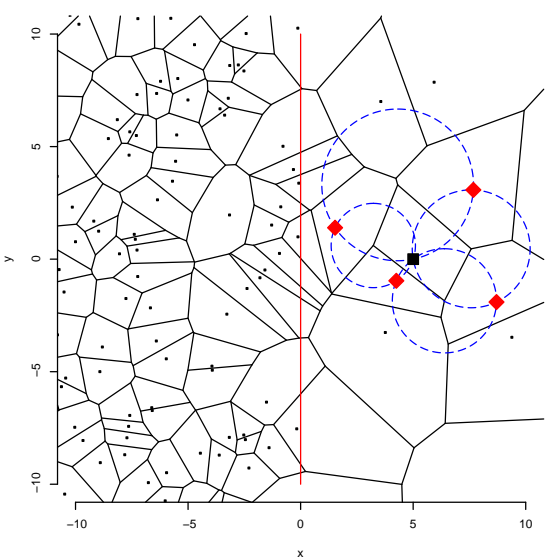

(a)

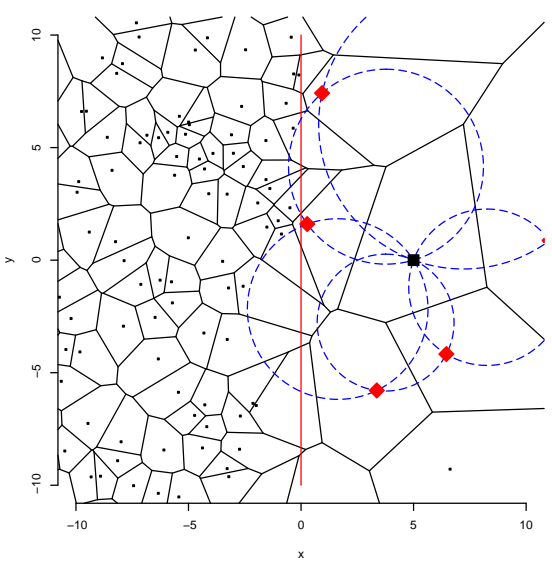

(b)

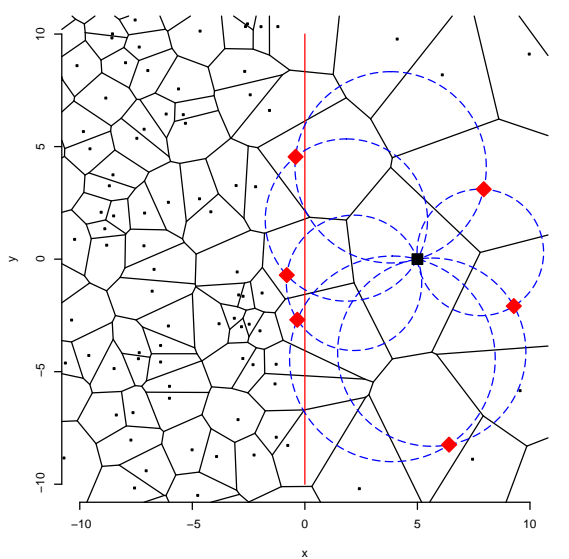

(c)

Figure 5: Fundamental region of the cell with the nucleus located at (5,0); (a) Any modification of the pattern of the nuclei left to the vertical line $x=0$ cannot modify this cell. (b) Any thinning of the pattern of the nuclei left to the vertical line $x=0$ cannot modify this cell. (c) A thinning or adding points to the pattern of the nuclei left to the vertical line $x=0$ can modify this cell.

such that $V\left(x, \Phi_{\chi}\right) \neq V\left(x, \Phi_{u}\right)$. The identification of such cells is the basis of all further error approximations presented in this section.

For a given point $x \in \mathbb{R}^{2}$ and a realization $\phi$ of a point process on $\mathbb{R}^{2}$, let $\mathcal{N}(x, \phi)$ denote the subset of points of $\phi$, which in the Voronoi tessellation $\mathcal{V}(\phi)$ have cells sharing an edge with the cell $V(x, \phi)$. Formally

$$
\mathcal{N}(x, \phi)=\{y \in \phi: \phi(B(x, y, z))=0 \text { for some } z \in \phi, x, y, z \text { distinct }\} .
$$

where $B(x, y, z)$ is the ball circumscribed on the points $x, y, z$. We have used above the well known principle, saying that three given elements from the set of the nuclei generating a VT share a common vertex if and only if the ball circumscribed on them does not contain in its interior any other nucleus. The union of empty balls $B(x, y, z)$ appearing in the definition of $\mathcal{N}(x, \phi)$ is called the fundamental region (or the Voronoi flower) of the cell $V(x, \phi)$ (see Figure 5). Note that the cell $V(x, \phi)$ preserves its shape when the pattern of nuclei $\phi$ is subject to changes only outside the fundamental region of $V(x, \phi)$. Thus, a cell $V\left(x, \Phi_{\chi}\right)$ with its nucleus $x \in \chi_{u}$ for some $u=1, \ldots, \ell$, can be different from $V\left(x, \Phi_{u}\right)$ if the fundamental region of $V\left(x, \Phi_{\chi}\right)$ is not totally contained in in $\chi_{u}$.

Verifying an inclusion property for the whole fundamental region is not an easy task. It is much easier to verify simply, for a nucleus $x \in \chi_{u}$, whether at least one of its neigbours in $\mathcal{N}(x, \phi)$ is outside $\chi_{u}$. Note however that it might be the case that all the neighbours $\mathcal{N}(x, \phi)$ are in $\chi_{u}$, and that the fundamental region of $V(x, \phi)$ intersects $\chi_{u}^{c}$. Then a modification of the pattern $\phi$ in the intersection of $\chi_{u}^{c}$ with the fundamental region of $V(x, \phi)$ might modify the shape of the cell $V(x, \phi)$. However any thinning (removing of points) of $\phi$ in $\chi_{u}^{c}$ will not modify the fundamental region of the cell $V(x, \phi)$ and consequently will not modify $V(x, \phi)$ itself.

This let us treat a cell $V\left(x, \Phi_{\chi}\right)$, with $x \in \chi_{u}$ for some $u=1, \ldots, \ell$, as possibly being different than $V\left(x, \Phi_{u}\right)$ only if at least one of its neigbours in $\mathcal{N}\left(x, \Phi_{u}^{\max }\right)$ is outside $\chi_{u}$, where $\Phi_{u}^{\max }$ is some auxiliary pattern that has more points than $\Phi_{\chi}$ and $\Phi_{u}$, and from whom both can be retrieved by thinning of points in $\chi_{u}^{c}$. 
More precisely, for each $u=1, \ldots, \ell$, we define

$$
\Phi_{u}^{\max }=\sum_{u^{\prime}: \lambda_{u^{\prime}} \geq \lambda_{u}} \sum_{i} \mathbb{I}\left(X_{i}^{u^{\prime}} \in \chi_{u^{\prime}}\right) \varepsilon_{X_{i}^{u^{\prime}}}+\sum_{u^{\prime \prime}: \lambda_{u^{\prime \prime}}<\lambda_{u}} \sum_{i} \mathbb{I}\left(X_{i}^{u} \in \chi_{u^{\prime \prime}}\right) \varepsilon_{X_{i}^{u}} .
$$

For a given set $A$, define

$$
\zeta(A, \phi)=\left\{x \in A: \mathcal{N}(x, \phi) \cap A^{c} \neq \emptyset\right\} ;
$$

i.e., a subset of $A$, such that if we place a nucleus in $\zeta(A, \phi)$, then this nucleus will have at least one neighbour among $\phi$ outside $A$. For $x \in \chi_{u}$, it is possible that $V\left(x, \Phi_{\chi}\right) \neq V\left(x, \Phi_{u}\right)$ only if $x \in \zeta\left(\chi_{u}, \Phi_{u}^{\max }\right)$. This statement will be formally justified in the proof of Proposition 4.1. Note that $\Phi_{u}^{\max }$ is a modulated Poisson process with intensity not less than $\lambda_{u}$. More precisely, it has the same intensity as $\Phi_{\chi}$ except the regions where the original intensity lower than $\lambda_{u}$ is raised to $\lambda_{u}$.

\subsection{Fixed modulation}

Let us denote by $\mathcal{M}_{u}$ the distribution of the typical cell of the homogeneous $\operatorname{PVT} \mathcal{V}\left(\Phi_{u}\right)$, $u=1, \ldots, \ell$. Formally, $\mathcal{M}_{u}$ is a measure on the space of closed subsets of $\mathbb{R}^{2}$ endowed with a suitable topology and the Borel $\sigma$-field $\mathbb{M}$ generated by it (see [9] for the measure-theoretic settings that are not explicitly used here, and [13] for more details).

The following result gives an approximation of the distribution of the typical cell in a fixed modulation scenario (see [9]).

Proposition 4.1 Let $\chi$-mod $P P \Phi_{\chi}$ be given, fix $x$ in the interior of $\chi_{u}$ for some $u \in\{1, \ldots, \ell\}$. Then the distribution of the cell $V\left(x, \Phi_{\chi}\right)-x$ can be approximated in total variation by the distribution $\mathcal{M}_{u}$ of the typical cell $V\left(0, \Phi_{u}\right)$ of the homogeneous PVT with the following error:

$$
\sup _{L \in \mathbb{M}}\left|\mathbf{P}\left(V\left(x, \Phi_{\chi}\right)-x \in L\right)-\mathcal{M}_{u}(L)\right| \leq \mathbf{P}\left(x \in \zeta\left(\chi_{u}, \Phi_{u}^{\max }\right)\right) .
$$

We recall the idea of the proof from [9] because it will be used later on.

Proof: Note first that we can take a probability space such that almost surely $\Phi_{u_{1}} \subset \Phi_{u_{2}} \subset$ $\ldots \subset \Phi_{u_{\ell}}$, where $\lambda_{u_{1}} \leq \lambda_{u_{2}} \leq \ldots \leq \lambda_{u_{\ell}}$. In fact, the distribution of the $\Phi_{\chi}$ remains the same if all $\Phi_{u}$ are constructed by successive thinning of $\Phi_{u_{\ell}}$. Let it be the case. Obviously

$$
\sup _{L \in \mathbb{M}}\left|\mathbf{P}\left(V\left(x, \Phi_{\chi}\right)-x \in L\right)-\mathcal{M}_{u}(L)\right| \leq \mathbf{P}\left(V\left(x, \Phi_{\chi}\right) \neq V\left(x, \Phi_{u}\right)\right) .
$$

Note now that $V\left(x, \Phi_{\chi}\right)=V\left(x, \Phi_{u}\right)$ is equivalent to $\mathcal{N}\left(x, \Phi_{\chi}\right)=\mathcal{N}\left(x, \Phi_{u}\right)$. Moreover, for $x \in$ $\chi_{u}$, if $\mathcal{N}\left(x, \Phi_{u}^{\max }\right) \subset \chi_{u}$, then $\mathcal{N}\left(x, \Phi_{u}^{\max }\right)=\mathcal{N}\left(x, \Phi_{\chi}\right)=\mathcal{N}\left(x, \Phi_{u}\right)$. Thus $V\left(x, \Phi_{\chi}\right) \neq V\left(x, \Phi_{u}\right)$ implies $\mathcal{N}\left(x, \Phi_{u}^{\max }\right) \not \subset \chi_{u}$; i.e., $x \in \zeta\left(\chi_{u}, \Phi_{u}^{\max }\right)$, which completes the proof.

The following result proved in [9] gives a numerically tractable upper bound of the righthand-side (rhs) of (4.1). Let us denote $\lambda_{\max }=\max \left\{\lambda_{u}: u=1, \ldots, \ell\right\}, \lambda_{\min }=\min \left\{\lambda_{u}: u=\right.$ $1, \ldots, \ell\}$.

Proposition 4.2 Let $x \in \chi_{u}$ and $\Phi_{\chi}$ be given as in Proposition 4.1. We have

$$
\mathbf{P}\left(x \in \zeta\left(\chi_{u}, \Phi_{u}^{\max }\right)\right) \leq 4 \lambda_{\max } \sum_{u^{\prime} \neq u} \max \left(\lambda_{u}, \lambda_{u^{\prime}}\right) \int_{\chi_{u^{\prime}}} e^{-\lambda_{u} \pi|x-y|^{2} / 8} \mathrm{~d} y .
$$

$\mathrm{RR} \mathrm{n}^{\circ} 5323$ 
Now we will consider mean functionals of the typical cell. Note that by Proposition 4.1 we can bound the approximation error for bounded functionals only. Consider now (possibly unbounded) non-negative functional $\Psi(V)$ of the cell $V$, which is translation invariant; i.e., $\Psi(V)=\Psi(V-x)$ for all $x$ (these two assumptions are from now on taken implicitly), and that satisfies the following property

$$
\Psi(V) \leq A(\mathcal{R}(V))^{\alpha}
$$

where $A, \alpha$ are some positive constants and $\mathcal{R}(V)$ is the minimal radius of the disc centered at the nucleus $x$ of $V$, that covers $V$; i.e.,

$$
\mathcal{R}(V)=\inf \left\{r: V \subset B_{r}(x)\right\}
$$

where $B_{r}(x)=\left\{z \in \mathbb{R}^{2}:|z-x|<r\right\}$. Note that all the examples of cell functionals presented in Section 3.1.3 satisfy condition (4.3).

In addition to $\Phi_{u}^{\max }$, let us define $\Phi^{\min }=\Phi_{u_{\min }}$, where $u_{\min }=\min \arg \left\{\lambda_{u}: u=1, \ldots, \ell\right\}$. The following result gives an approximation of the mean functional of the typical cell in the fixed modulation scenario.

Proposition 4.3 Let $x \in \chi_{u}$ and $\Phi_{\chi}$ be given as in Proposition 4.1. Suppose a cell functional $\Psi(\cdot)$ satisfies condition (4.3). Then its mean of the cell $V\left(x, \Phi_{\chi}\right)$ can be approximated by the respective mean of the typical cell $V\left(\Phi_{u}\right)$ of the homogeneous PVT with the following error:

$$
\left|\mathbf{E}\left[\Psi\left(V\left(x, \Phi_{\chi}\right)\right)\right]-\mathbf{E}\left[\Psi\left(V\left(0, \Phi_{u}\right)\right)\right]\right| \leq A \mathbf{E}\left[\mathbb{I}\left(x \in \zeta\left(\chi_{u}, \Phi_{u}^{\max }\right)\right)\left(\mathcal{R}\left(V\left(x, \Phi^{\min }\right)\right)\right)^{\alpha}\right] .
$$

Proof: Using the same arguments as in the proof of Proposition 4.1 we find that

$$
\begin{aligned}
& \left|\mathbf{E}\left[\Psi\left(V\left(x, \Phi_{\chi}\right)\right)\right]-\mathbf{E}\left[\Psi\left(V\left(0, \Phi_{u}\right)\right)\right]\right| \\
& \quad \leq \mathbf{E}\left[\mathbb{1}\left(x \in \zeta\left(\chi_{u}, \Phi_{u}^{\max }\right)\right)\left|\Psi\left(V\left(x, \Phi_{\chi}\right)\right)-\Psi\left(V\left(x, \Phi_{u}\right)\right)\right|\right] .
\end{aligned}
$$

Moreover, since $\Psi$ is non-negative, and by condition (4.3),

$$
\begin{aligned}
\left|\Psi\left(V\left(x, \Phi_{\chi}\right)\right)-\Psi\left(V\left(x, \Phi_{u}\right)\right)\right| & \leq \max \left(\Psi\left(V\left(x, \Phi_{\chi}\right)\right), \Psi\left(V\left(x, \Phi_{u}\right)\right)\right) \\
& \leq A\left(\max \left(\mathcal{R}\left(V\left(x, \Phi_{\chi}\right)\right), \mathcal{R}\left(V\left(x, \Phi_{u}\right)\right)\right)\right)^{\alpha} \\
& \leq A\left(\mathcal{R}\left(V\left(x, \Phi^{\mathrm{min}}\right)\right)\right)^{\alpha},
\end{aligned}
$$

where the last inequality follows from the fact that $\mathcal{R}(V(x, \phi))$ increases when the pattern of points $\phi$ is thinned. This completes the proof.

Denote by

$$
\mathcal{R}(y, z)=\sqrt{\frac{|y-z|}{4 \sin ^{2}(\arg (y)-\arg (z))}}
$$

the radius of the disc circumscribed on points $0, x, y \in \mathbb{R}^{2}$. The following result gives a numerically tractable upper bound of the right-hand-side (rhs) of (4.4). 
Proposition 4.4 Let $x \in \chi_{u}$ and $\Phi_{\chi}, \Psi$ be given as in Proposition 4.3. Then

$$
\begin{aligned}
& \mathbf{E}\left[\mathbb{I}\left(x \in \zeta\left(\chi_{u}, \Phi_{u}^{\max }\right)\right)\left(\mathcal{R}\left(V\left(x, \Phi^{\min }\right)\right)\right)^{\alpha}\right] \\
& \leq \lambda_{\max }^{2} \int_{\chi_{u}^{c}} \int_{\mathbb{R}^{2}} e^{-\lambda_{u} \pi \mathcal{R}^{2}(y-x, z-x)} \\
& \quad \times 6\left((2 \mathcal{R}(y-x, z-x))^{\alpha}+\frac{\alpha e^{\frac{2}{3} \pi \lambda_{\min } R^{2}(y-x, z-x)} \Gamma\left(\frac{\alpha}{2}, \frac{2}{3} \pi \lambda_{\min } R^{2}(y-x, z-x)\right)}{2\left(\frac{\pi \lambda_{\min }}{6}\right)^{\frac{\alpha}{2}}}\right) \mathrm{d} z \mathrm{~d} y,
\end{aligned}
$$

where $\Gamma(\alpha, a)=\int_{a}^{\infty} e^{-t} t^{\alpha-1} \mathrm{~d} t$.

Proof: Note that by the definition

$$
\mathbb{I}\left(x \in \zeta\left(\chi, \Phi_{u}^{\max }\right)\right)=\mathbb{I}\left(\mathcal{N}\left(x, \Phi_{u}^{\max }\right) \cap \chi_{u}^{c} \neq \emptyset\right)
$$

and for $x \in \chi_{u}$

$$
\begin{aligned}
& \mathbb{I}\left(\mathcal{N}\left(x, \Phi_{u}^{\max }\right) \cap \chi_{u}^{c} \neq \emptyset\right) \leq \#\left(\mathcal{N}\left(x, \Phi_{u}^{\max }\right) \cap \chi_{u}^{c}\right) \\
& \quad \leq \frac{1}{2} \int_{\mathbb{R}^{2}} \mathbb{I}\left(y \in \chi_{u}^{c}\right) \int_{\mathbb{R}^{2}} \mathbb{I}\left(\Phi_{u}^{\max }(B(x, y, z))=0\right) \mathbb{I}(y \neq z) \Phi_{u}^{\max }(\mathrm{d} z) \Phi_{u}^{\max }(\mathrm{d} y)
\end{aligned}
$$

where $1 / 2$ stands in the previous bound since each point $y \in \mathcal{N}\left(x, \Phi_{u}^{\max }\right)$, as a neighbour of $x$, is counted by the integral there with 2 different co-neighbours $z$. Thus, by the Slivnyak's theorem

$$
\begin{aligned}
& \mathbf{E}\left[\mathbb{I}\left(x \in \zeta\left(\chi_{u}, \Phi_{u}^{\max }\right)\right)\left(\mathcal{R}\left(V\left(x, \Phi^{\min }\right)\right)\right)^{\alpha}\right] \\
& \leq \frac{\lambda_{\max }^{2}}{2} \int_{\chi_{u}^{c}} \int_{\mathbb{R}^{2}} \mathbf{E}\left[\mathbb{I}\left(\Phi_{u}(B(x, y, z))=0\right)\left(\mathcal{R}\left(V\left(x, \Phi^{\min }\right)\right)\right)^{\alpha}\right] \mathrm{d} z \mathrm{~d} y .
\end{aligned}
$$

Now,

$$
\begin{aligned}
& \mathbf{E}\left[\mathbb{I}\left(\Phi_{u}(B(x, y, z))=0\right)\left(\mathcal{R}\left(V\left(x, \Phi^{\mathrm{min}}\right)\right)\right)^{\alpha}\right] \\
& \leq \mathbf{P}\left(\Phi_{u}(B(x, y, z))=0\right) \mathbf{E}\left[\left(\mathcal{R}\left(V\left(x, \Phi^{\mathrm{min}}\right)\right)\right)^{\alpha} \mid \Phi_{u}(B(x, y, z))=0\right] \\
& =e^{-\lambda_{u} \pi \mathcal{R}^{2}(y-x, z-x)} \mathbf{E}\left[\left(\mathcal{R}\left(V\left(0, \Phi^{\mathrm{min}}\right)\right)\right)^{\alpha} \mid \Phi_{\min }(B(0, y-x, z-x))=0\right] .
\end{aligned}
$$

The result follows from Lemma A.1 that is proved in the appendix.

More explicit bounds are presented in the Appendix.

\subsection{Random modulation}

Consider now a stationary modulating random partition $\Xi=\left\{\Xi_{u}: u=1, \ldots, \ell\right\}$ be independent of Poisson processes $\Phi_{u}$ and the resulting stationary-Cox Voronoi tessellation (CoxVT) $\mathcal{V}\left(\Phi_{\Xi}\right)$. Let $p_{u}=\mathbf{P}\left(0 \in \Xi_{u}\right)$. Note that $p_{u}$ is the fraction of the plane covered by $\Xi_{u}$ and so is called the volume fraction of $\Xi_{u}$.

The following proposition gives an approximation of the distribution of the typical cell of $\Xi$-mod PVT by a linear combination of the distributions $\mathcal{M}_{u}$ of the typical cells in the homogeneous scenarios. 
Proposition 4.5 The distribution $\mathcal{M}_{(\Xi)}$ of the typical cell of the $\Xi$-mod $P V T \mathcal{V}\left(\Phi_{\Xi}\right)$ admits the following decomposition

$$
\mathcal{M}_{(\Xi)}(L)=\frac{1}{\lambda_{(\Xi)}} \sum_{u=1}^{\ell} \lambda_{u} p_{u} \mathcal{M}_{u}(L)+R
$$

where $\lambda_{(\Xi)}=\sum_{u=1}^{\ell} \lambda_{u} p_{u}$ and the remainder term is bounded by

$$
|R| \leq \frac{1}{\lambda_{(\Xi)}} \sum_{u=1}^{\ell} \lambda_{u} \mathbf{P}\left(0 \in \zeta\left(\Xi_{u}, \Phi_{u}^{\max }\right)\right) .
$$

Proof: The distribution of the typical cell of a stationary double stochastic Poisson point process is given by the following general formula

$$
\mathcal{M}_{(\Xi)}(L)=\frac{1}{\lambda_{(\Xi)}} \sum_{u=1}^{\ell} E\left[\mathbb{I}\left(0 \in \Xi_{u}\right) \mathbb{I}\left(V\left(0, \Phi_{\Xi}\right) \in L\right)\right] .
$$

Now

$$
\begin{aligned}
\mathbf{E} & {\left[\mathbb{I}\left(0 \in \Xi_{u}\right) \mathbb{I}\left(V\left(0, \Phi_{\Xi}\right) \in L\right)\right] } \\
& =p_{u} \mathcal{M}_{u}(L)+\mathbf{E}\left[\mathbb{I}\left(0 \in \Xi_{u}\right)\left(\mathbb{I}\left(V\left(0, \Phi_{\Xi}\right) \in L\right)-\mathbb{I}\left(V\left(0, \Phi_{u}\right) \in L\right)\right)\right]
\end{aligned}
$$

and using Proposition 4.1, given a realization of $\Xi$, we get

$$
\mathbf{E}\left[\mathbb{I}\left(0 \in \Xi_{u}\right)\left|\mathbb{I}\left(V\left(0, \Phi_{\Xi}\right) \in L\right)-\mathbb{I}\left(V\left(0, \Phi_{u}\right) \in L\right)\right|\right] \leq \mathbf{E}\left[\mathbb{I}\left(0 \in \zeta\left(\Xi_{u}, \Phi_{u}^{\max }\right)\right)\right],
$$

because, by the definition of $\zeta, 0 \in \zeta\left(\Xi_{u}, \Phi_{u}^{\max }\right)$ implies $0 \in \Xi_{u}$. This completes the proof.

In order to better express the quality of the above approximation, for each $u=1 \ldots, \ell$ denote by $C_{u}(x)$ the so-called covariance function of the random set $\Xi_{u}$ defined by

$$
C_{u}(x)=\mathbf{P}\left(0 \in \Xi_{u}, x \in \Xi_{u}\right) .
$$

Note that $C_{u}(x)$ is the probability that both points 0 and $x$ simultaneously belong to $\Xi_{u}$. This probability is known explicitly for some simple models, including the Boolean model and can be estimated from a given observed set.

The following result gives a numerically tractable upper bound of the rhs of (4.6).

Proposition 4.6 Let $\Phi_{\Xi}$ be given as in Proposition 4.5. We have

$$
\mathbf{P}\left(0 \in \zeta\left(\Xi_{u}, \Phi_{u}^{\max }\right)\right) \leq 4 \lambda_{\max }^{2} \int_{\mathbb{R}^{2}}\left(p_{u}-C_{u}(y)\right) e^{-\lambda_{u} \pi|y|^{2} / 8} \mathrm{~d} y
$$

Proof: For a given realization of $\Xi$ we use Proposition 4.2 and get

$$
\mathbf{P}\left(0 \in \zeta\left(\Xi_{u}, \Phi_{u}^{\max }\right)\right) \leq 4 \lambda_{\max }^{2} \int_{\mathbb{R}^{2}} \mathbf{E}\left[\mathbb{I}\left(0 \in \Xi_{u}\right) \mathbb{I}\left(y \notin \Xi_{u}\right)\right] e^{-\lambda_{u} \pi|y|^{2} / 8} \mathrm{~d} y .
$$

Note now that

$$
\mathbf{P}\left(0 \in \Xi_{u}, y \notin \Xi_{u}\right)=\mathbf{P}\left(0 \in \Xi_{u}\right)-\mathbf{P}\left(0 \in \Xi_{u}, y \in \Xi_{u}\right),
$$

which completes the proof. 
Consider now a non-negative, translation invariant functional $\Psi(V)$ of the cell $V$, which satisfies condition (4.3). The following result gives an approximation of the mean functional of the typical cell of the CoxVT.

Proposition 4.7 Let $\Phi_{\Xi}$ be given as in Proposition 4.5. Suppose a cell functional $\Psi(\cdot)$ satisfies condition (4.3). Then its mean of the typical cell of the $\Xi-\bmod P V T \mathcal{V}\left(\Phi_{\Xi}\right)$ can be approximated by the following linear combination of the respective means in the homogeneous PVT's

$$
\int \Psi(V) \mathcal{M}_{(\Xi)}(\mathrm{d} V)=\frac{1}{\lambda_{(\Xi)}} \sum_{u=1}^{\ell} \lambda_{u} p_{u} \mathbf{E}\left[\Psi\left(V\left(0, \Phi_{u}\right)\right)\right]+R
$$

where the remainder term is bounded by

$$
|R| \leq \frac{A}{\lambda_{(\Xi)}} \sum_{u=1}^{\ell} \lambda_{u} \mathbf{E}\left[\mathbb{I}\left(0 \in \zeta\left(\Xi_{u}, \Phi_{u}^{\max }\right)\right)\left(\mathcal{R}\left(V\left(0, \Phi^{\min }\right)\right)\right)^{\alpha}\right]
$$

Proof: Follow the same lines as in the proofs of Propositions 4.5 and 4.3 .

The following result gives a numerically tractable upper bound of the rhs of (4.9).

Proposition 4.8 Let $\Phi_{\Xi}$ and $\Psi$ be given as in Proposition 4.7. We have

$$
\begin{aligned}
& \mathbf{E}\left[\mathbb{I}\left(0 \in \zeta\left(\Xi_{u}, \Phi_{u}^{\max }\right)\right)\left(\mathcal{R}\left(V\left(0, \Phi^{\min }\right)\right)\right)^{\alpha}\right] \\
& \leq \frac{\lambda_{\max }^{2}}{\lambda_{u}^{\frac{\alpha}{2}+2}} \int_{\mathbb{R}^{2}} \int_{\mathbb{R}^{2}}\left(p_{u}-C_{u}\left(\frac{y}{\lambda_{u}}\right)\right) e^{-\pi \mathcal{R}^{2}(y, z)} \\
& \quad \times 6\left((2 \mathcal{R}(y, z))^{\alpha}+\frac{\alpha e^{\frac{2}{3} \pi \frac{\lambda_{\min }}{\lambda_{u}} \mathcal{R}^{2}(y, z)} \Gamma\left(\frac{\alpha}{2}, \frac{2}{3} \pi \frac{\lambda_{\min }}{\lambda_{u}} R^{2}(y, z)\right)}{2\left(\frac{\pi \lambda_{\min }}{6 \lambda_{u}}\right)^{\frac{\alpha}{2}}}\right) \mathrm{d} z \mathrm{~d} y .
\end{aligned}
$$

Proof: It follows from Proposition 4.8; use the same arguments as in the proof of Proposition 4.6. The factor $\lambda_{u}^{-\alpha / 2-2}$ can be obtained using the following scaling property of the Poisson process: the dilation by some factor $\gamma>0$ of all point of the Poisson process with intensity $\lambda$ on $\mathbb{R}^{2}$ leads to the Poisson process with intensity $\lambda / \gamma^{2}$.

The double integral in the upper bound given in Proposition 4.8 can be further simplified when the covariance function $C_{u}(y)$ is rotation invariant function of $y$. The following result follows from Lemma A.1 and Lemma A.2 in the Appendix.

Corollary 4.9 If $C_{u}(y)=\bar{C}_{u}(|y|)$ is rotation invariant, then

$$
\begin{aligned}
& \mathbf{E}\left[\mathbb{I}\left(0 \in \zeta\left(\Xi_{u}, \Phi_{u}^{\max }\right)\right)\left(\mathcal{R}\left(V\left(0, \Phi^{\min }\right)\right)\right)^{\alpha}\right] \\
& \leq \frac{2 \pi \lambda_{\max }^{2}}{\lambda_{u}^{\alpha / 2+2}} \int_{0}^{\infty}\left(p_{u}-\bar{C}_{u}\left(\frac{t}{\sqrt{\lambda_{u}}}\right)\right) e^{-\frac{\pi t^{2}}{4}} \mathcal{W}_{\alpha, \frac{\lambda_{\min }}{\lambda_{u}}}(t) \mathrm{d} t
\end{aligned}
$$

where

$$
\begin{aligned}
\mathcal{W}_{\alpha, \lambda}(t)= & \left(3+\frac{\pi}{2}\right) \mathcal{D}_{1}(\alpha) \mathcal{K}(\alpha) t^{\alpha+2} \\
& +\left(3+\frac{\pi}{2}\right) \mathcal{D}_{2}(\alpha, \lambda) \mathcal{K}(\alpha-2) t^{\alpha} \\
& +\left(3+\frac{\pi}{2}\right) \mathcal{D}_{3}(\alpha, \lambda) \mathcal{K}(0) t^{2} \\
& +\left(3+\frac{\pi}{2}\right)\left(\mathcal{D}_{1}(\alpha) \mathcal{K}(\alpha)+\mathcal{D}_{2}(\alpha, \lambda) \mathcal{K}(\alpha-2)+\mathcal{D}_{3}(\alpha, \lambda) K(0)\right)
\end{aligned}
$$

and the functions $\mathcal{D}_{i}$ and $\mathcal{K}$ are given, respectively in Lemma A.1 and Lemma A.2.

$\mathrm{RR} \mathrm{n}^{\circ} 5323$ 


\section{Discussion and numerical examples}

In this section we will summarize the mathematical results presented in the previous section and discuss their relevance to modeling of the architecture of communication networks. We will show numerical examples as well.

It was explained in Section 2 that the modeling of a particular deterministic architecture of a network by a random homogeneous-Poisson structure allows for catching various "homogeneous irregularities" of the existing network in a statistical way; i.e., by a small number of essential parameters, the main being planar constant densities of the network elements. What if these irregularities in reality seem not to be homogeneous? We can distinguish two cases:

- regions of the deviation from the constant density are well identified, of a relatively simple geometry; moreover, we want to analyze a part of the network (say modeled by a PVT cell) that lies in the "homogeneously irregular" part, in some distance to the deviations of the density. In this case we can use fixed modulation of the density; examples of this scenarios are simple "hot spot" and "hot wall" described in Section 3.2.3.

- regions of different values of the density are irregularly scattered, with this irregularity begin homogeneous but on a much larger scale than the irregularity of the repartition of the network elements. Then, we can try to catch both the local irregularities of the repartition of the network elements and the global irregularities of the density of this repartition in a statistical way. In this case we propose the model with a random modulation of the density. A simple, analytically tractable example of this scenario is the modulation via a Boolean model (see Section 3.2.3).

For a scenario with a fixed modulation of the density, the result of Propositions 4.5 and 4.8 can be rephrased as follows.

Corollary 5.1 The value of a mean functional $\Psi$ of the cell $V\left(x, \Phi_{\chi}\right)$ located in the region $\chi_{u}$, where the density is constant $\lambda_{u}$, in some distance to other regions $\chi_{u^{\prime}}, u^{\prime} \neq u$, where the density might deviate from $\lambda_{u}$, can be approximately taken as that of the typical cell of the homogeneous PVT with the density $\lambda_{u}$ (thus ignoring all the density deviations)

$$
\mathbf{E}\left[\Psi\left(V\left(x, \Phi_{\chi}\right)\right)\right] \approx \mathbf{E}\left[\Psi\left(x, \Phi_{u}\right)\right]
$$

Propositions 4.2 and 4.4 are supposed to quantify the error of the above approximation that is obviously a decreasing function of the distance of the cell nucleus $x$ to the regions of the deviation of the density.

For a scenario with a random modulation of the density, the result of Propositions 4.8 and 4.8 can be rephrased as follows.

Corollary 5.2 The value of a mean functional $\Psi$ of a typical cell $V$ (say located at 0 ) of a homogeneous "doubly irregular" (double-stochastic Poisson) Voronoi tessellation can be approximated by a linear combination of its values for the respective "simple" homogeneous PVT's

$$
\mathbf{E}\left[\Psi\left(V\left(0, \Psi_{\Xi}\right)\right)\right] \approx \frac{1}{\lambda_{(\Xi)}} \sum_{u=1}^{\ell} \lambda_{u} p_{u} \mathbf{E}\left[\Psi\left(V\left(0, \Phi_{u}\right)\right)\right]
$$

with the coefficients $\lambda_{u} p_{u}$ interpreted as the fraction of the overall density $\Lambda_{(\Xi)}$ of the nuclei due to regions of the local density $\lambda_{u}$. 
Propositions 4.6, 4.8 and Corollary 4.9 are supposed to quantify the error of the above approximation that is decreasing in the difference between the scale of global irregularities of the density and the scale of the local repartition density of the network elements. We will illustrate this in the following example.

Example 5.3 Consider an inhomogeneous Poisson Voronoi tessellation, where the density of the Poisson process is equal to $\lambda_{1}$ in the Boolean model $\Xi$ (see Section 3.2.3) and $\lambda_{2}$ in its complement $\Xi^{c}$. Suppose that the grains of the BM $\Xi$ (i.e., the regions of the density $\lambda_{1}$ ) are spherical, with random iid radii $\rho_{i}$, and that the density of these regions is equal to $\beta$. It is known (see Section 3.1.1 in [17]) that the volume fraction and the covariance function of the $\mathrm{BM} \Xi$ are equal to, respectively,

$$
p_{1}=p=\mathbf{P}(0 \in \Xi)=1-e^{-\beta \pi \mathbf{E}\left[\rho^{2}\right]}
$$

and

$$
\bar{C}_{1}(r)=\bar{C}(r)=\mathbf{P}(0 \in \Xi, x \in \Xi \text { with }|x|=r)=(1-p)-(1-p)^{2} e^{\beta \pi \mathbf{E}[L(\rho, r)]} .
$$

where $L(r)$ is the surface area of the lune created by the set-difference of the two discs with radii $\rho$ each, mutually shifted by $r, L(\rho, r)=\left|B_{\rho}((0,0))-B_{\rho}((0, r))\right|$. Thus

$$
L(\rho, r)= \begin{cases}\rho^{2}\left(\pi-2 \arccos \left(\frac{r}{2 \rho}\right)+\frac{1}{2} r \sqrt{4 \rho^{2}-r^{2}} \leq\left(\frac{\pi}{2}+1\right) r \rho\right. & \text { if } r \leq 2 \rho \\ \pi \rho^{2} & \text { if } r>2 \rho\end{cases}
$$

Consequently

$$
p_{1}-C_{1}(r)=(1-p)\left(1-e^{-\beta \mathbf{E}[L(\rho, r)]}\right) \leq \begin{cases}(1-p)\left(1-e^{-\beta(\pi / 2+1) r \mathbf{E}[\rho]}\right) & \text { if } r \leq 2 \rho \\ p(1-p) & \text { if } r>2 \rho\end{cases}
$$

Note by the symmetry that for the complement $\Xi^{c}$ of the BM

$$
p_{2}-\bar{C}_{2}(r)=\mathbf{P}(0 \notin \Xi, x \in \Xi \text {, with }|x|=r)=\mathbf{P}(0 \in \Xi, x \notin \Xi \text {, with }|x|=r)=p-\bar{C}(r) .
$$

Applying the rhs of (5.2) to the inequality established in Corollary 4.9 we see that the error of the approximation of (5.1) tends to 0 when $\beta \rightarrow 0$ and $E\left[\rho^{2}\right] \rightarrow \infty$ such that $\beta \mathbf{E}\left[\rho^{2}\right]=-\log (p) / \pi=$ const; that is, when the spots of the density $\lambda_{1}$ become large and sparsely distributed with the fraction $p$ of the plane covered by this density constant.

Consider now two functionals of the typical cell of our inhomogeneous PVT. Let $\Psi_{1}(V)=$ $|\partial V|$ be the perimeter of the cell $V=V(0, \phi)$ with the nucleus at the origin 0 and let $\Psi_{2}(V)=$ $\int_{V}|x| \mathrm{d} x$ (see Section 3.1.3 for possible interpretation of these functionals). Note that both functionals satisfy condition (4.3). Indeed, $\Psi_{1}(V) \leq 2 \pi \mathcal{R}(V)$ and $\Psi_{2}(V) \leq \frac{2 \pi}{3} \mathcal{R}^{3}(V)$. For a homogeneous PVT with density $\lambda$ the mean values of these functionals are known explicitly; $\mathbf{E}\left[\Psi_{1}(V)\right]=4 \lambda^{-1 / 2}$ and $\mathbf{E}\left[\Psi_{2}(V)\right]=1 / 2 \lambda^{-3 / 2}$ (see Section 3.1.4). Using Corollary 5.1 we can approximate their mean values for our inhomogeneous PVT as follows

$$
\begin{aligned}
& \left.\mathbf{E}\left[\Psi_{1}\left(V\left(0, \Psi_{\Xi}\right)\right)\right] \approx \frac{4}{\lambda_{(\Xi)}}\left(\sqrt{\lambda_{1}} p+\sqrt{\lambda_{2}}(1-p)\right)\right), \\
& \mathbf{E}\left[\Psi_{2}\left(V\left(0, \Psi_{\Xi}\right)\right)\right] \approx \frac{1}{2 \lambda_{(\Xi)}}\left(\frac{p}{\sqrt{\lambda_{1}}}+\frac{1-p}{\sqrt{\lambda_{2}}}\right)
\end{aligned}
$$

$\mathrm{RR} \mathrm{n}^{\circ} 5323$ 


\begin{tabular}{|c|c|c|}
\hline$\kappa$ & error in (5.3) in \% & error in $(5.4)$ in $\%$ \\
\hline 3.00 & $0.1 \cdot 10^{-8}$ & $0.1 \cdot 10^{-5}$ \\
3.40 & & $0.5 \cdot 10^{-4}$ \\
3.50 & & $0.1 \cdot 10^{-3}$ \\
3.55 & & $0.2 \cdot 10^{-3}$ \\
3.56 & & $0.2 \cdot 10^{-3}$ \\
3.57 & $0.1 \cdot 10^{-6}$ & 13515.3 \\
4.00 & $0.2 \cdot 10^{-5}$ & 14306.1 \\
4.58 & $0.5 \cdot 10^{-4}$ & \\
4.59 & $0.5 \cdot 10^{-4}$ & \\
4.60 & 218.5 & \\
4.75 & 222.0 & \\
5.00 & 227.8 & 15994.5 \\
\hline
\end{tabular}

Table 1: Relative error bounds in \% of the approximations (5.3) and (5.4).

where $\lambda_{(\Xi)}=\lambda_{1} p+\lambda_{2}(1-p)$. In order to analyze numerically the errors of the above approximations, let us take for example $\lambda_{1}=4, \lambda_{2}=40, \beta=\kappa \cdot 10^{-7}$, constant $\rho=70 \sqrt{30 / \kappa}$. For these values (for any value of $\kappa>0$ ) we have the fraction of the surface covered by the BM equal to $p=0.04513$, average density $\lambda_{(\Xi)}=5.625$ and

$$
\begin{aligned}
\mathbf{E}\left[\Psi_{1}\left(V\left(0, \Phi_{1}\right)\right)\right]=25.2982, & \mathbf{E}\left[\Psi_{1}\left(V\left(0, \Phi_{2}\right)\right)\right]=8.0000 \\
\mathbf{E}\left[\Psi_{1}\left(V\left(0, \Phi_{\Xi}\right)\right)\right] \approx & 8.7807 \\
\mathbf{E}\left[\Psi_{2}\left(V\left(0, \Phi_{1}\right)\right)\right]=0.7981, & \mathbf{E}\left[\Psi_{2}\left(V\left(0, \Phi_{2}\right)\right)\right]=0.2500 \\
\mathbf{E}\left[\Psi_{2}\left(V\left(0, \Phi_{\Xi}\right)\right)\right] \approx & 0.2423 .
\end{aligned}
$$

The approximation errors depend on $\kappa$ and are presented in Table 1. We observe an interesting cutoff phenomenon for these approximation errors. They stay negligible for the density $\beta$ of spots of the BM below some threshold and explode for $\beta$ above it. We conjecture that this cutoff is not only due to various bounds we made throughout the analysis, but it reflects a similar cutoff of the "exact error", which might hold however for a different (smaller) value of $\beta$. Note that the similar observation follows from numerical examples presented in [9].

\section{Appendix}

We will prove now the following technical lemma, that is used in the proof of Proposition 4.4. We will also give some further bounds for the integrals under consideration. Recall that $\mathcal{R}(y, z)$ denotes the radius of the disc circumscribed on points $0, x, y \in \mathbb{R}^{2}$. 
Lemma A.1 Let $\Phi$ be a Poisson point process with intensity $\lambda$ and consider the radius $\mathcal{R}(V(0, \Phi))$ of the minimal disc centered at 0 that covers the cell $V(0, \Phi)$. Then for $\alpha \geq 1$

$$
\begin{aligned}
& \mathbf{E}\left[(\mathcal{R}(V(0, \Phi)))^{\alpha} \mid \Phi(B(0, y, z))=0\right] \\
& \leq 6\left((2 \mathcal{R}(y, z))^{\alpha}+\frac{\alpha e^{\frac{2}{3} \pi \lambda R^{2}(y, z)} \Gamma\left(\frac{\alpha}{2}, \frac{2}{3} \pi \lambda \mathcal{R}^{2}(y, z)\right)}{2\left(\frac{\pi \lambda}{6}\right)^{\frac{\alpha}{2}}}\right) \\
& \leq D_{1}(\alpha) R^{\alpha}+D_{2}(\alpha, \lambda) \mathcal{R}^{\alpha-2}(y, z)+D_{3}(\alpha, \lambda)
\end{aligned}
$$

where $D_{1}(\alpha)=3 \cdot 2^{\alpha+1}, D_{2}(\alpha, \lambda)=3\left(\frac{2}{3}\right)^{\alpha / 2-1} \alpha e \Gamma\left(\frac{\alpha}{2}\right)\left(\frac{6}{\lambda \pi}\right)^{\alpha / 2}, D_{3}(\alpha, \lambda)=3 \alpha e \Gamma\left(\frac{\alpha}{2}\right)\left(\frac{6}{\lambda \pi}\right)^{\alpha / 2}$ and $\Gamma(\alpha)=\Gamma(\alpha, 0)$.

Proof: Denote by $\mathcal{C}$ the event $\{\Phi(B(0, y, z))=0\}$. Note that radius $\mathcal{R}(V(0, \Phi))$ of the minimal disc centered at 0 that covers the cell $V(0, \Phi)$ can only increase when we enlarge the region where $\Phi$ does not have points. Thus

$$
\mathbf{E}\left[\mathcal{R}^{\alpha}(V(0, \Phi)) \mid \mathcal{C}\right] \leq \mathbf{E}\left[\mathcal{R}^{\alpha}(V(0, \Phi)) \mid \mathcal{C}^{\prime}\right]
$$

where

$$
\mathcal{C}^{\prime}=\left\{\Phi\left(B_{0}(2 \mathcal{R}(y, z))\right)=0\right\}
$$

recall that $B_{0}(r)$ is the disc in $\mathbb{R}^{2}$ centered at 0 , with radius $r$. Now, note that

$$
\mathcal{R}(V(0, \Phi)) \leq \max \left(R_{i}: i=1, \ldots, 6\right)
$$

where $R_{i}, i=1, \ldots, 6$ are the nearest to the origin 0 points of $\Phi$ sitting respectively in six angular sectors of with 60 degree each, stemming from the origin. Thus

$$
\mathbf{E}\left[\mathcal{R}^{\alpha}(V(0, \Phi)) \mid \mathcal{C}^{\prime}\right] \leq \mathbf{E}\left[\max \left(R_{i}^{\alpha}: i=1, \ldots, 6\right) \mid \mathcal{C}^{\prime}\right] \leq 6 \mathbf{E}\left[R_{1}^{\alpha} \mid \mathcal{C}^{\prime}\right]
$$

since $R_{i} i=1, \ldots, 6$ have the same distribution under $\mathcal{C}^{\prime}$ (they are also independent but this property is not used here)

$$
\mathbf{P}\left(R_{i}>t \mid \mathcal{C}^{\prime}\right)= \begin{cases}1 & \text { if } t \leq 2 \mathcal{R}(y, z), \\ e^{-\lambda \pi / 6\left(t^{2}-4 \mathcal{R}^{2}(y, z)\right)} & \text { if } t>2 \mathcal{R}(y, z) .\end{cases}
$$

Thus for $\alpha \geq 1$

$$
\begin{aligned}
\mathbf{E}\left[\mathcal{R}^{\alpha}(V(0, \Phi)) \mid \mathcal{C}\right] & \leq 6 \alpha \int_{0}^{\infty} t^{\alpha-1} \mathbf{P}\left(R_{1}>t \mid \mathcal{C}^{\prime}\right) \mathrm{d} t \\
& =6\left((2 \mathcal{R}(y, z))^{\alpha}+\frac{\alpha e^{\frac{2}{3} \pi \lambda \mathcal{R}^{2}(y, z)} \Gamma\left(\frac{\alpha}{2}, \frac{2}{3} \pi \lambda \mathcal{R}^{2}(y, z)\right)}{2\left(\frac{\pi \lambda}{6}\right)^{\frac{\alpha}{2}}}\right)
\end{aligned}
$$

The second inequality in Lemma A.1 follows from the following observation: for $K>0, \eta>0$

$$
\Gamma(\eta, K)=e^{-K} \int_{0}^{\infty} e^{-t}(t+K)^{\eta-1} \mathrm{~d} t \leq e^{-K} e \Gamma(\eta)\left(1+K^{\eta-1}\right) .
$$


Lemma A.2 We have for $\alpha \in \mathbb{R}$, and $0 \neq y \in \mathbb{R}^{2}$

$$
\int_{\mathbb{R}^{2}} e^{-\pi \mathcal{R}^{2}(y, z)} \mathcal{R}^{\alpha} \mathrm{d} z=I_{1}-I_{2}+I_{3}+I_{4},
$$

where

$$
\begin{aligned}
& I_{1}=\int_{|y| / 2}^{\infty} e^{-\pi s^{2}} s^{\alpha-1}|y|^{3} \frac{1}{\sqrt{4 s^{2}-|y|^{2}}} \mathrm{~d} s, \\
& I_{2}=-\int_{|y| / 2}^{\infty} e^{-\pi s^{2}} s^{\alpha+1} \arcsin \left(\frac{|y|}{2 s}\right) \mathrm{d} s \leq 0, \\
& I_{3}=\int_{|y| / 2}^{\infty} e^{-\pi s^{2}} s^{\alpha-1}|y| \sqrt{4 s^{2}-|y|^{2}} \mathrm{~d} s, \\
& I_{4}=\int_{|y| / 2}^{\infty} e^{-\pi s^{2}} s^{\alpha+1} \mathrm{~d} s .
\end{aligned}
$$

Moreover

$$
\begin{aligned}
& |y| I_{1}=2^{-\alpha}|y|^{4} e^{-\frac{\pi|y|^{2}}{4}} \int_{0}^{\infty} e^{-\frac{\pi s^{2}}{4}}\left(s^{2}+|y|^{2}\right)^{\frac{\alpha}{2}-1} \mathrm{~d} s \leq 2 \mathcal{K}(\alpha)\left(1+|y|^{\alpha+2}\right) e^{\frac{-\pi|y|^{2}}{4}}, \\
& |y| I_{3}=2^{-\alpha}|y|^{2} e^{-\frac{\pi|y|^{2}}{4}} \int_{0}^{\infty} e^{-\frac{\pi s^{2}}{4}}\left(s^{2}+|y|^{2}\right)^{\frac{\alpha}{2}-1} s^{2} \mathrm{~d} s \leq \mathcal{K}(\alpha)\left(1+|y|^{\alpha+2}\right) e^{\frac{-\pi|y|^{2}}{4}} \\
& |y| I_{4}=2^{-\alpha-1} \pi|y| e^{-\frac{\pi|y|^{2}}{4}} \int_{0}^{\infty} e^{-\frac{\pi s^{2}}{4}}\left(s^{2}+|y|^{2}\right)^{\frac{\alpha}{2}} s \mathrm{~d} s \leq \frac{\pi}{2} \mathcal{K}(\alpha)\left(1+|y|^{\alpha+2}\right) e^{\frac{-\pi|y|^{2}}{4}},
\end{aligned}
$$

where

$$
\mathcal{K}(\alpha)=\left(\frac{\pi}{4}\right)^{-\frac{\alpha}{2}-\frac{3}{2}} \Gamma\left(\frac{\alpha}{2}+\frac{3}{2}, 2\right) .
$$

Proof: The expression (A.1) follows from

$$
\int_{\mathbb{R}^{2}} \ldots \mathrm{d} z=2 \int_{0}^{\pi} \int_{0}^{\infty} \ldots r \mathrm{~d} r \mathrm{~d} \theta
$$

and the substitution

$$
\begin{cases}r=\left|s \cos \theta+\sqrt{4 s^{2}-|y|^{2}} \sin \theta\right| & \text { for } s \geq|y| / 2, \\ \theta=\theta & 0 \leq \theta \pi\end{cases}
$$

whose Jacobian is equal to $4 s \sin \theta\left(4 s^{2}-|y|^{2}\right)^{-1 / 2}$. The inequalities (A.6)-(A.7) follow from the following observation: for $a, K>0 \eta \geq 0$,

$$
\int_{0}^{\infty} e^{-a s^{2}}(s+K)^{\eta} \mathrm{d} s \leq\left(1+K^{\eta}\right) 2^{\eta-1} a^{-\frac{1}{2}-\frac{\eta}{2}} \Gamma\left(\frac{\eta}{2}+\frac{1}{2}, \sqrt{\frac{\pi}{a}}\right) .
$$

\section{Acknowledgments}

This research has been partly supported by KBN grant 2 P03A 02023. 


\section{References}

[1] F. Baccelli and B. Blaszczyszyn. On a coverage process ranging from the Boolean model to the Poisson-Voronoi tessellation, with applications to wireless communications. Adv. Appl. Prob., 33:293-323, 2001.

[2] F. Baccelli, B. Błaszczyszyn, and P. Mühlethaler. An Aloha protocol for multihop mobile wireless networks. In Proc. of ITC Specialist Seminar on Performance Evaluation of Wireless and Mobile Systems, Antwerp, Belgium, 2004.

[3] F. Baccelli, B. Błaszczyszyn, and F. Tournois. Spatial averages of coverage characteristics in large CDMA networks. Wireless Networks, 8:569-586, 2002.

[4] F. Baccelli, B. Błaszczyszyn, and F. Tournois. Downlink capacity and admission/congestion control in CDMA networks. In Proc. of IEEE INFOCOM, San Francisco, 2003.

[5] F. Baccelli, M. Klein, M. Lebourges, and S. Zuyev. Géométrie aléatoire et architecture de réseaux de communication. Annales des Télécommunications, 51:158-179, 1996. (In French).

[6] F. Baccelli, M. Klein, M. Lebourges, and S. Zuyev. Stochastic geometry and architecture of communication networks. Telecommunications systems, 7:209-227, 1997.

[7] F. Baccelli, D. Kofman, and J. L. Rougier. Self organizing hierarchical multicast trees and their optimization. In Proceedings of IEEE INFOCOM, New York (USA), 1999.

[8] F. Baccelli and S. Zuyev. Stochastic geometry models of mobile communication networks. In J.H. Dshalalow, editor, Frontiers in queueing. Models and Applications in Science and Engineering, pages 227-244. CRC Press, Boca Raton, 1997.

[9] B. Błaszczyszyn and R. Schott. Approximate decomposition of some modulated-Poisson Voronoi tessellations. Adv. in Appl. Probab., 35:847-862, 2003.

[10] P.J. Courtois. Decomposability, Queueing and Computer System Applications. Academic Press, New York, New York, 1977.

[11] R. Cowan. Properties of ergodic random mosaic processes. Math. Nachr., 97:89-102, 1980.

[12] S. Farinetto and S. Zuyev. Stochastic geometry modeling of hybrid optical networks. Performance Evaluations, 2004. to appear.

[13] G. Matheron. Random Sets and Integral Geometry. John Willey \& Sons, London, 1975.

[14] J. Mecke. Palm methods for stationary random mosaics. In R.V. Ambartzumian, editor, Combinatorial Principles in Stochastic Geometry, pages 124-132. Armenian Academy of Sciences Publ., 1980.

[15] J. Møller. Lectures on Random Voronoi Tessellations, volume 87 of Lecture Notes in Statistics. Springer-Verlag, New York, 1989. 
[16] A. Okabe, B. Boots, and K. Sugihara. Spatial Tessellations: Concepts and Applications of Voronoi Diagrams. John Willey \& Sons, Chichester, 2000.

[17] D. Stoyan, W. Kendall, and J. Mecke. Stochastic Geometry and its Applications. Wiley, Chichester, 1995. 


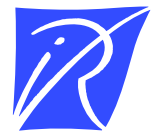

Unité de recherche INRIA Rocquencourt Domaine de Voluceau - Rocquencourt - BP 105 - 78153 Le Chesnay Cedex (France)

Unité de recherche INRIA Lorraine : LORIA, Technopôle de Nancy-Brabois - Campus scientifique 615, rue du Jardin Botanique - BP 101 - 54602 Villers-lès-Nancy Cedex (France)

Unité de recherche INRIA Rennes : IRISA, Campus universitaire de Beaulieu - 35042 Rennes Cedex (France)

Unité de recherche INRIA Rhône-Alpes : 655, avenue de 1'Europe - 38330 Montbonnot-St-Martin (France)

Unité de recherche INRIA Sophia Antipolis : 2004, route des Lucioles - BP 93 - 06902 Sophia Antipolis Cedex (France)

INRIA - Domaine de Voluceau - Rocquencourt, BP 105 - 78153 Le Chesnay Cedex (France)

http://www.inria.fr

ISSN 0249-6399 